\title{
Current status of long term ventilation of children in the United Kingdom: questionnaire survey
}

\author{
E Jardine, M O'Toole, J Y Paton, C Wallis
}

\begin{abstract}
Objectives To identify the number and current location of children, aged 0 to 16 years, requiring long term ventilation in the United Kingdom, and to establish their underlying diagnoses and ventilatory needs.

Design Postal questionnaires sent to consultant respiratory paediatricians and all lead clinicians of intensive care and special care baby units in the United Kingdom.

Subjects All children in the United Kingdom who, when medically stable, continue to need a mechanical aid for breathing.
\end{abstract}

Results 141 children requiring long term ventilation were identified from the initial questionnaire. Detailed information was then obtained on 136 children from 30 units. Thirty three children $(24 \%)$ required continuous positive pressure ventilation by tracheostomy over 24 hours, and 103 received ventilation when asleep by a non-invasive mask $(\mathrm{n}=62 ; 46 \%)$, tracheostomy $(\mathrm{n}=32 ; 24 \%)$, or negative pressure ventilation $(n=9 ; 7 \%)$. Underlying conditions included neuromuscular disease $(n=62$; $46 \%$ ), congenital central hypoventilation syndrome $(\mathrm{n}=18 ; 13 \%)$, spinal injury $(\mathrm{n}=16 ; 12 \%)$, craniofacial syndromes $(\mathrm{n}=9 ; 7 \%)$, bronchopulmonary dysplasia $(\mathrm{n}=6 ; 4 \%)$, and others $(\mathrm{n}=25 ; 18 \%) .93$ children were cared for at home. 43 children remained in hospital because of home circumstances, inadequate funding, or lack of provision of home carers. 96 children were of school age and 43 were attending mainstream school.

Conclusions A significant increase in the number of children requiring long term ventilation in the United Kingdom has occurred over the past decade.

Contributing factors include improved technology, developments in paediatric non-invasive ventilatory support, and a change in attitude towards home care. Successful discharge home and return to school is occurring even for severely disabled patients. Funding and home carers are common obstacles to discharge.

\section{Introduction}

Society has the new responsibility of considering those children who are dependent on technology for their survival. A growing population of children exist who have chronic respiratory failure due to conditions such as muscle disease, disorders of ventilatory control, or abnormalities of the airway, and who require long term ventilation. ${ }^{12}$ At present these children often experience prolonged hospitalisations, which separate them from their families and place them in environments inappropriate for their health and development. A recent survey of intensive care units showed that there were significant numbers of children requiring long term ventilation who were resident in hospital, often in an intensive care setting. ${ }^{3}$ It is recognised that hospital is both an unsuitable environment for a growing child and an inappropriate use of resources. ${ }^{4}$ Concerns have also been raised that the continuing availability of paediatric intensive care beds would be severely curtailed unless successful home transfer of these children was achieved. ${ }^{1}$

The benefits of home care of children requiring long term ventilation are well documented. ${ }^{5-7}$ Advances in ventilator technology, and a growing experience and acceptance of home care, have increased the possibilities for discharging children requiring long term ventilation from hospital to home. Ten years ago a survey identified 24 children in the United Kingdom on long term ventilation, nine of whom were cared for at home. ${ }^{8}$ In 1993, a postal survey of the Paediatric Intensive Care Society identified 31 children receiving ventilatory support at home. ${ }^{9}$ Unfortunately, the response rate to the questionnaire was poor. Subsequently there have been no data on the status of home care of children requiring long term ventilation in the United Kingdom, despite considerable advances in the field and ongoing concerns regarding funding and care in the community.

To obtain up to date information on the current use of long term ventilation in children, we surveyed all consultant respiratory paediatricians and the lead clinicians of intensive care units and special care baby units about children ventilated long term for respiratory failure. Such information is central to the planning of resource allocation, and for evaluating the discharge process and enabling further study into the impact of home ventilation on the children, their families, and the health service.

\section{Subjects and methods}

Initially we sent a brief questionnaire to all UK consultant respiratory paediatricians and lead clinicians of intensive care and special care baby units asking them to identify the number of children aged 0 to 16 years who were dependent on long term ventilatory support.

We defined long term ventilatory support as: "any child who, when medically stable, continued to need a mechanical aid for breathing which may be acknowledged after a failure to wean, three months after the institution of ventilation." Medically stable was not defined but was left to the judgment of the child's consultant. We excluded data on premature infants who were likely to wean successfully, but included data on children with chronic lung disease of prematurity where weaning was proving impossible beyond three months from term.

We obtained contact addresses for all 112 consultant members of the British Paediatric Respiratory Society from the society's secretary. Of the 112 initial questionnaires posted, $89 \quad(80 \%)$ consultants responded. In addition, we obtained the addresses of the 49 lead clinicians of paediatric intensive care units

\author{
Institute of Child \\ Health and Great \\ Ormond Street \\ Hospital for \\ Children NHS \\ Trust, London \\ WC1N 3JH \\ E Jardine, \\ research sister \\ M O'Toole, \\ social worker \\ C Wallis, \\ consultant \\ Department of \\ Child Health, \\ Yorkhill, Glasgow \\ G3 8SJ \\ J Y Paton, \\ senior lecturer \\ Correspondence to: \\ Dr Wallis \\ c.wallis@ich.ucl.ac.uk
}

BMJ 1999;318:295-9 
from the Paediatric Intensive Care Society, of whom 30 $(61 \%)$ responded to our initial questionnaire. All nonresponders or their secretaries were contacted by telephone, and the number of children on long term ventilation was noted. We also became aware of a few children through the parents of other children requiring long term ventilatory support. We then contacted the relevant hospital in the area.

We established a working party of 20 healthcare workers from centres throughout the United Kingdom who had a specific interest in the care of children on long term ventilation. This network provided us with a back up to ensure maximal ascertainment of cases.

The children were registered by their surname, first name, date of birth, and home postcode. Only six children were reported twice by different consultants. These children were registered once on the database under the lead consultant.

All positive responders were sent a more detailed questionnaire. The specific information requested on each child included:

- Date of birth and home postcode

- The diagnostic category most responsible for ventilator dependence (for example neuromuscular disease)

- Date of onset of ventilation (month and year)

- The present location of the child-that is, hospital unit or home

- Time in present location (in months)

- Reasons for hospital stay, if applicable

- Method of ventilatory support

- Time dependent on the ventilator

- Type of ventilator device and maintenance

- Professionals coordinating the discharge

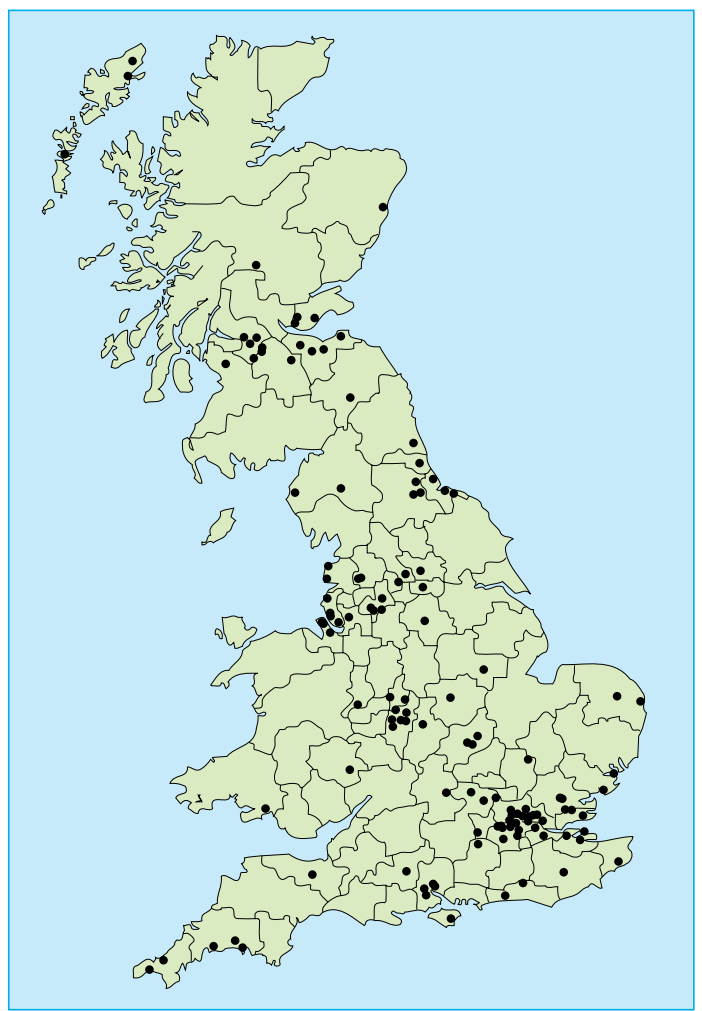

Geographical distribution of 136 children requiring long term ventilation in the United Kingdom
Table 1 Range of specialties caring for 136 children on long term ventilation in the United Kingdom

\begin{tabular}{lc} 
Consultant specialty & No of children \\
\hline Respiratory paediatrician & 65 \\
\hline Chest physician & 19 \\
\hline General paediatrician & 15 \\
\hline Spinal injury & 10 \\
\hline Anaesthetist & 7 \\
\hline Paediatric intensivist & 7 \\
\hline Paediatric neurologist & 5 \\
\hline Paediatric neurosurgeon & 2 \\
\hline Neurodevelopmental paediatrician & 2 \\
\hline Paediatric anaesthetist & 1 \\
\hline ENT (paediatric) & 1 \\
\hline Anaesthetics/spinal injury (adult) & 1 \\
\hline Plastic surgeon (paediatric) & 1 \\
\hline
\end{tabular}

Table 2 Number of children on long term ventilation in the United Kingdom according to age

\begin{tabular}{lr} 
Age interval & No \\
\hline$<12$ months & 5 \\
\hline 1 to 5 years & 44 \\
\hline 6 to 10 years & 43 \\
\hline 11 to 16 years & 44
\end{tabular}

- Educational provision

- Concurrent use of other technology (for example oxygen treatment and monitoring, transcutaneous carbon dioxide monitoring, humidification, wheelchair use)

- Funding sources

- Support at home.

We reviewed the birth dates and home postcodes to ensure that no child was included twice. Replies were then entered on to a database.

\section{Results}

From the initial questionnaire, 141 children requiring long term ventilatory support were identified. More detailed questionnaires were then sent out in May 1997. We received replies from 30 centres, with 49 primary consultants identified from a wide range of specialities (table 1). The survey documented 136 children in the United Kingdom who were chronically dependent on a mechanical aid to breathe (figure). We failed to gain full details on five children despite repeated requests.

\section{Age range and aetiology}

Table 2 lists the number of children requiring long term ventilation in the United Kingdom according to age range. The main aetiologies of children requiring long term ventilatory support were neuromuscular disease, congenital central hypoventilation syndrome (Ondine's Curse), spinal injury, craniofacial syndromes, and bronchopulmonary dysplasia (table 3 ).

Ninety three children $(68 \%)$ were cared for at home. Table 4 details the locations of the children in hospital. In each category more children were cared for at home than in hospital. The only exception was bronchopulmonary dysplasia, when five out of six children received hospital care. 
Table 3 Main diagnostic categories of children requiring long term ventilation in the United Kingdom

\begin{tabular}{lcc} 
Disorder & $\begin{array}{c}\text { No at } \\
\text { home }\end{array}$ & $\begin{array}{c}\text { No in } \\
\text { hospital }\end{array}$ \\
\hline Neuromuscular disease & 52 & 10 \\
\hline Congenital central hypoventilation syndrome & 13 & 5 \\
\hline Spinal injury & 10 & 6 \\
\hline Craniofacial syndrome & 9 & 0 \\
\hline Bronchopulmonary dysplasia & 1 & 5 \\
\hline Other & 8 & 17 \\
\hline Total & 93 & 43 \\
\hline
\end{tabular}

Table 4 Hospital location of 43 children requiring long term ventilation in the United Kingdom

\begin{tabular}{lc} 
Hospital location & No of children \\
\hline General ward & 17 \\
\hline Step down unit* & 10 \\
\hline Intensive care unit & 7 \\
\hline Spinal injury unit & 3 \\
\hline Special care baby unit & 3 \\
\hline Community home & 3 \\
\hline
\end{tabular}

${ }^{*}$ Any unit that is not classified as either a paediatric intensive care unit or ward setting - for example, high dependency unit, transitional care unit, long term ventilation unit.

Table 5 Types of ventilatory support used at home and in hospital to treat 136 children requiring long term ventilation in the United Kingdom

\begin{tabular}{lcc} 
Ventilatory support & Home & Hospital \\
\hline Delivery mode & & \\
\hline Tracheostomy & 33 & 32 \\
\hline Non-invasive mask & 52 & 10 \\
\hline Negative pressure device & 8 & 1 \\
\hline
\end{tabular}

Table 6 Obstacles to discharge in 43 hospitalised children requiring long term ventilation

Obstacle No of children

\begin{tabular}{lc}
\hline Staff unavailable for support at home & 22 \\
\hline Funding negotiations not complete & 16 \\
\hline Housing unsuitable & 15 \\
\hline Not established on ventilator for home use & 12 \\
\hline Social reasons & 9 \\
\hline Awaiting transfer to local hospital & 7
\end{tabular}

\section{Mode of ventilatory support}

Sixty five children (48\%) received positive pressure support by a tracheostomy. Sixty two children (46\%) needed ventilation by a face or nasal mask, and nine children $(7 \%)$ were managed with negative pressure ventilation.

Fifty two of the 93 children $(56 \%)$ at home used non-invasive ventilation by a face or nasal mask. This reflects the high proportion of children with neuromuscular disease who are commonly ventilated using non-invasive techniques (table 5).

\section{Time on ventilatory support}

Children who were dependent on ventilatory support fell into three broad categories: those on support 24 hours per day; those receiving support only when asleep; and those receiving support intermittently when awake and when asleep.

Thirty three of the 136 children (24\%) required ventilatory support 24 hours per day. Of these, $13 \mathrm{had}$ been successfully discharged home. Ninety six children $(71 \%)$ required ventilation when asleep only; 20 were still in hospital, and three were in a paediatric intensive care unit. Seven children required additional ventilatory support intermittently during the day as well as when asleep.

\section{Reasons for prolonged hospitalisation}

Several factors were identified as delaying discharge from hospital to home (table 6). Twenty two children $(51 \%)$ remained in hospital due to failure to recruit qualified nursing staff or trained carers to care for the child in the home environment. Incomplete funding arrangements were a factor in $16(37 \%)$ cases. A further 15 children $(35 \%)$ were still cared for in hospital because their home was assessed as unsuitable to meet either the child's needs or that of the equipment.

\section{Funding of home care}

Ventilator equipment for the home care of eight children $(9 \%)$ was provided either by the parents or from charitable sources. Only one child received the full cost of home care from the Department of Social Services. The remaining 84 children $(90 \%)$ received funding for their respiratory equipment and support from the local health authority.

\section{Patient support at home}

The skill of healthcare professionals assisting families in the care of the children at home varied widely. Forty six families (49\%) met all their child's medical needs themselves. Despite this burden on the families, only 16 $(17 \%)$ had formal respite arrangements in place.

\section{Education}

The most surprising finding was the number of children attending mainstream schools. Of 81 children of school age requiring long term ventilation and living at home with their families, 43 (53\%) attended mainstream schools. Only two of the 81 children required a home tutor. This was because their local mainstream school was unable to cope with their disability, and because attendance at the local special needs school would have involved a 100 mile round trip. Thirty six children attended special needs schools.

Of the 15 children of school age still in hospital, only three attended the hospital school. The other 12 children attended schools in the community, with three going to their previous mainstream school on a daily basis.

\section{Discussion}

Our survey of long term ventilation of children in the United Kingdom, completed during 1997, docu-

Table 7 Incidence of children in the United Kingdom dependent on ventilatory support by calendar year

\begin{tabular}{lcccccccccc} 
& 1988 & 1989 & 1990 & 1991 & 1992 & 1993 & 1994 & 1995 & 1996 & $1997^{*}$ \\
\hline Incidence & 1 & 2 & 4 & 4 & 11 & 19 & 11 & 27 & 28 & 18 \\
\hline
\end{tabular}

*Until end of September. 
ments a significant increase in the prevalence of children requiring long term ventilatory support since Robinson first reported on this subject in $1990,{ }^{8}$ and a rising incidence (table 7).

From the outset, we aimed for complete ascertainment by identifying and contacting all centres and consultants in the United Kingdom who were likely to care for children requiring long term ventilatory support. The initial questionnaire identified 141 such children. Of this group, detailed information was reported in 136 cases. While our findings may not include all cases, they do provide the best available figures for this important group of children. Factors contributing to these increased numbers include improved technology, developments in non-invasive ventilatory support of children and its use in neuromuscular disease, a change in attitude towards home care of these children, and an increase in the number of survivors of paediatric and neonatal intensive care units. ${ }^{10}$

A recent survey of children requiring long term ventilatory support in Canada identified 82 such children. ${ }^{11}$ This number is comparable per head of population to our study. In France, the number of children dependent on long term ventilation, and the underlying aetiologies, are also comparable to our study. ${ }^{12}$ Although the United States has not fully studied this area, it does show a similar pattern, except for a uniquely large number of infants with bronchopulmonary dysplasia. In the United States, considerable cost savings have been shown in ventilated children who have returned home safely on a comprehensively planned programme of care..$^{13}$

Our study has established that children dependent on long term ventilatory support are successfully being reintegrated into their homes and returning to school, even in the case of severe disability. The number of children successfully discharged from hospital to home, despite the need for ongoing technological care, was greater than anticipated. A previous report highlighted the inappropriateness of caring for long term ventilated children in intensive care. ${ }^{3}$ Our current findings, however, show that a shift of care seems to have occurred from intensive care units to units where stabilisation of the medical condition, rehabilitation, and reintegration are achieved. This is an appropriate but difficult move requiring effort, time, and resources. For those children still in hospital, the main problems were neither medical nor technical; discharge was frequently stalled by the emotional and practical support required and limited resources.

A surprising and encouraging finding in our study was that most of the children attended mainstream schools. However, there was great disparity in the type of education available to the cohort. Four children requiring ventilatory support 24 hours per day were educated in mainstream schools, yet 26 children with less physical disability and dependent only on ventilatory support during the night were educated in special needs schools.

We identified only nine children for whom family problems were listed as a cause of delayed discharge from hospital. On further investigation, four of the children were expected to return home eventually and the other five were thought to be unable to return home. All these children required ventilation 24 hours
- The number of children requiring long term ventilatory support has increased substantially in the past 8 years

- Ventilatory support at home is the best option for meeting the child's psychological needs and enhancing quality of life

- The majority of children dependent on long term ventilation live at home and attend mainstream schools

- A shift of care has occurred from intensive care units to less acute areas

per day. Four of the five children had learning disabilities in addition to their physical disabilities. In two cases, the family withdrew from any involvement, and the children remain hospitalised awaiting fostering.

The discharge process can be long and complicated. To help with this issue, discharge guidelines have been formulated by a working party on long term ventilation of children in the United Kingdom. ${ }^{14}$ The key principles of the discharge process are that the child is a member of the family, that the family must be supported and involved in decision making, and that the responsibility for care of the child transfers from the hospital to the community health team and the family at discharge. The guidelines emphasise the importance of communication and teamwork.

Currently, the financial burden of home care falls on the health authority in which the child resides, as there is no centralised funding for individuals dependent on ventilatory support. Not surprisingly, funding for home ventilation is a common obstacle to discharge and can take many months of negotiation to arrange. Our study established that considerable variability exists between health authorities with respect to the funding requirements of children dependent on ventilatory support. Costs do differ widely depending on the level of dependency of the child and associated disabilities. ${ }^{14}$

Home care for most children requiring long term ventilatory support is the best option for meeting the child's medical and psychological needs. It also enhances their quality of life. It is likely that this population of children will continue to increase as medical technology advances and a better understanding of the role of ventilatory support at home develops. Having established minimum prevalence figures there is a need for continuing data on appropriate outcome measures, clinical progress, and incidence figures to assist the rational planning of services.

We thank the staff who responded to our survey and I Barker, B Boosfeld, R Bullock, C Doyle, S Kerr, R Lane, K Lucking, I Noyes, H Porter, M Samuels, J Shneerson, A Simonds, A Walker, T Ward, J W H Watt, and R Yates as members for the UK working party on the long term ventilation of children.

Contributors: CW and MO'T conceived the study, secured the funding, and wrote the paper. EJ coordinated the survey, collected the data under the supervision of CW and JYP, and wrote the paper. EJ will act as guarantor for the paper.

Funding: NHS Executive, Research and Development Directorate, and Mother and Child Health Programme.

Conflict of interest: None. 
1 Fraser J, Henrichsen T, Mok Q, Tasker R. Prolonged mechanical ventilation as a consequence of acute illness. Arch Dis Child 1998;78:253-6.

2 Simonds AK. From intensive care unit to home discharge in the 24 hour ventilator-dependent patient. Eur Respir Mon 1998;8:364-79.

3 Fraser J, Mok Q, Tasker R. Survey of occupancy of paediatric intensive care units by children who are dependent on ventilators. BMJ 1997:315:347-8.

4 Davies RH, Harvey I, Newton-John H, Ward TA. Home ventilation of a child with motor and sensory neuropathy. BMJ 1996;313:153-5.

5 Frates RC, Splaingard ML, Smith EO, Harrison GM. Outcome of home mechanical ventilation. J Pediatr 1985;106:850-6.

6 Eigen $\mathrm{H}$, Zander J. Home mechanical ventilation of pediatric patients. Am Rev Respir Dis 1990;141:258-9.

7 Fields AI, Rosenblatt A, Pollack MM, Kaufman J. Home care cost-effectiveness for respiratory technology-dependent children. $\mathrm{Am} \mathrm{J}$ Dis Child 1991;145:729-33.

8 Robinson RO. Ventilator dependency in the United Kingdom. Arch Dis Child 1990;65:1235-6.
9 Barker I. Central funding and a central resource providing information are needed. BMJ 1996;313:940.

10 Newton-John HF. Long-term mechanical ventilation of patients in Australia. Med J Aust 1989;150:3-6.

11 Dhillon JS, Frewen TC, Singh NC, Speechley KN. Chronic mechanical ventilation-dependent children in Canada. Paediatr Child Health 1996;1:111-6.

12 Fauroux B, Howard P, Muir JF. Home treatment for chronic respiratory insufficiency: the situation in Europe in 1992. The European working group on home treatment for chronic respiratory insufficiency. Eur Respir J1994;7:1721-6

13 Goldberg AI, Faure AM, Vaughan CJ, Snarski R, Seleny FL. Home care for life-supported persons: an approach to program development. J Pediatr 1984;104:785-95.

14 Jardine E, Wallis C. Core guidelines for the discharge home of the child on long-term assisted ventilation in the United Kingdom. Thorax 1998;53:762-7.

(Accepted 9 November 1998)

\title{
Dilemmas in treating early prostate cancer: the evidence and a questionnaire survey of consultant urologists in the United Kingdom
}

\author{
Jenny L Donovan, Stephen J Frankel, Alex Faulkner, Sara Selley, David Gillatt, Freddie C Hamdy
}

Evidence based medicine suggests that evidence of effectiveness should accumulate, preferably from randomised controlled trials, before treatments for any condition become widely used. The case of localised prostate cancer shows how difficult this can be in practice. The suitability of population screening for localised prostate cancer has been debated, ${ }^{12}$ with particular concerns about the comparative effectiveness of the main treatments for the disease: radical prostatectomy, radical radiotherapy, and conservative management (also known as watchful waiting or surveillance). ${ }^{34}$ Systematic reviews show that published evidence is limited to two seriously flawed randomised controlled trials and a range of observational studies with biases relating to patient selection, variable treatment techniques, outcome assessments, and methods of data analysis. ${ }^{3}$ These studies show that 10 year survival is good and overlaps for the three treatments, being $85-90 \%$ for radical prostatectomy, $65-90 \%$ for radical radiotherapy, and $70-90 \%$ for conservative management. ${ }^{3}$ Although some studies indicate a survival advantage of radical treatments in some patients, this advantage is small and uncertain given the particular study designs. Furthermore, quality of life may be worse among those receiving radical treatments because of resulting complications. ${ }^{5}$ For example, after radical prostatectomy up to $3 \%$ of patients may be totally incontinent, with up to $60 \%$ "dribbling" urine, and 20-80\% impotent, while after radical radiotherapy up to $36 \%$ may have damage to adjacent organs, $10 \%$ incontinence, and 40\% impotence. ${ }^{3}$ Morbidity from conservative management relates to symptoms (and hormonal treatment if required) if the disease progresses.

\section{Subjects, methods, and results}

A postal questionnaire survey of practising consultant urologists registered with the British Association of Urological Surgeons was conducted exploring their treatment preferences for various clinical case vignettes. General surgeons, trainees, and those with paediatric caseloads were excluded. A total of 244 consultant urologists replied (response rate 60\%). Urologists had a mean of 14.1 years' experience (range 2-30 years), and 130 of them managed 100 patients or more with prostate cancer. Expertise in performing radical prostatectomy was restricted to comparatively few urologists-98 reported having ever performed the procedure and only $12(14 \%)$ that they performed 20 or more operations per year. The table shows that radical treatments were the first choice treatment for all hypothetical patients with apparently localised disease under the age of 70 years (cases 1, 2, 3, and 5 in table), irrespective of mode of presentation, prostate specific antigen concentration, and grade of tumour. Radical prostatectomy was preferred for the man of 55 (case 1), radical radiotherapy for the man of 69 (case 3). Conservative management was first choice for the majority of urologists for the man of 75 (case 4). For the youngest men (cases 1 and 5) only a few urologists selected conservative management, rising to $27 \%$ for the man aged 69.

\section{Comment}

Although reliable evidence supporting radical treatments is scarce, British urologists seem to favour them for all patients under 70 . These findings may be open to misinterpretation, representing what urologists say they do rather than what they do, although hospital episode statistics confirm that numbers of radical prostatectomies have doubled nearly every year between 1990-1 and 1994-5 in the United Kingdom (hospital episode statistics, 1989-90 to 1994-5).

Clearly, evidence is needed from randomised controlled trials, but such studies have proved difficult because of perceptions that patients are reluctant to accept conservative management. New methodological approaches are required urgently to investigate this
Editorial

by Emberton

Department of Social Medicine, University of Bristol, Canynge Hall, Bristol BS8 2PR

Jenny L Donovan, senior lecturer Stephen J Frankel, professor

Alex Faulkner, research associate

Sara Selley, research associate

Bristol Urological Institute,

Southmead

Hospital, Bristol

BS10 5NB

David Gillatt,

consultant urologist

University Urology

Unit, Freeman

Hospital, Newcastle

upon Tyne,

NE7 7DN

Freddie C Hamdy, consultant senior

lecturer

Correspondence to: Mr Hamdy

F.C.Hamdy@ncl.ac.uk

BMJ 1999;318:299-300 